ESJ Social Sciences

\title{
Metodología para Evaluación y Actualización de los Contenidos del Syllabus
}

\author{
Marcel Oswaldo Méndez-Mantuano
}

Magíster en Gestión Ambiental

Instituto Superior Tecnológico Juan Bautista Aguirre, Ecuador

Mariuxi Yomaira Olvera Morán

Magíster en Sistemas Integrados de Gestión

Instituto Superior Tecnológico Juan Bautista Aguirre, Ecuador

Evelyn Carolina Egüez Caviedes

Magíster en Dirección del Talento Humano

Instituto Superior Tecnológico Juan Bautista Aguirre, Ecuador

Keyla Ximena Bodero Jiménez

Magíster en Estadística Mención en Gestión de la Calidad y Productividad

Instituto Superior Tecnológico Juan Bautista Aguirre, Ecuador

\section{Darwin Vicente Apolo Robles}

Ingeniero Agroindustrial

Instituto Superior Tecnológico Juan Bautista Aguirre, Ecuador

Doi:10.19044/esj.2021.v17n32p90

Submitted: 24 July 2021

Accepted: 08 September 2021

Published: 30 September 2021
Copyright 2021 Author(s)

Under Creative Commons BY-NC-ND

4.0 OPEN ACCESS

Cite As:

Méndez-Mantuano M.O., Olvera Morán M.Y., Egüez Caviedes E.C., Bodero Jiménez K.X. \& Apolo Robles D.V. (2021). Metodología para Evaluación y Actualización de los Contenidos del Syllabus. European Scientific Journal, ESJ, 17 (32), 90.

https://doi.org/10.19044/esj.2021.v17n32p90

\section{Resumen}

Un buen contenido de Syllabus es una promesa que realiza el docente en beneficio de sus estudiantes. Por ello, existe la necesidad de valorar los temas que son expuestos en las diferentes cátedras, ya que los contextos sociales, económicos y productivos son dinámicos y evolucionan de manera constante y sostenida. La investigación propone una metodología para la evaluación y actualización de los contenidos de los Syllabus, a través de 4 diferentes perspectivas: docente, estudiantil, empresarial y tendencial, por medio del análisis de un estudio de caso aplicado para la asignatura de Matemática Aplicada de la carrera de Tecnología en Administración y dictada 
durante el segundo período académico 2020 - 2021 (diciembre a mayo). La propuesta metodológica se desarrolló por medio de encuestas a los grupos focales determinados (docentes, estudiantes y delegados empresariales), también se realizó un análisis del Syllabus de la asignatura de Matemática Aplicada a la Administración de la carrera de Licenciatura en Administración del Tecnológico Nacional de México y se contrastaron los contenidos del Syllabus en análisis y el perteneciente a la institución mexicana. Cada una de las 4 perspectivas tiene una valoración porcentual del $25 \%$ en relación con el promedio de los promedios en cada uno de los ítems. La semaforización de los resultados finales del estudio de caso determinó que el $65 \%$ de los contenidos (ítems) se deben mantener dentro de los Syllabus de Matemática Aplicada de la carrera de Tecnología en Administración, mientras que el $35 \%$ de dichos contenidos se tienen que reestructurar, de tal manera, que se fomente el desarrollo aplicativo de los contenidos dentro de un contexto laboralproductivo. También existen contenidos temáticos que deben ser incorporados dentro del documento pedagógico. La propuesta es flexible, donde se posibilita la incorporación de otros elementos que ayuden a esclarecer y fortalecer la metodología.

Palabras claves: Evaluación, metodología, syllabus 


\title{
Metodología para Evaluación y Actualización de los Contenidos del Syllabus
}

\author{
Marcel Oswaldo Méndez-Mantuano \\ Magíster en Gestión Ambiental \\ Instituto Superior Tecnológico Juan Bautista Aguirre, Ecuador \\ Mariuxi Yomaira Olvera Morán \\ Magíster en Sistemas Integrados de Gestión \\ Instituto Superior Tecnológico Juan Bautista Aguirre, Ecuador \\ Evelyn Carolina Egüez Caviedes \\ Magíster en Dirección del Talento Humano \\ Instituto Superior Tecnológico Juan Bautista Aguirre, Ecuador \\ Keyla Ximena Bodero Jiménez. \\ Magíster en Estadística Mención en Gestión de la Calidad y Productividad \\ Instituto Superior Tecnológico Juan Bautista Aguirre, Ecuador \\ Darwin Vicente Apolo Robles \\ Ingeniero Agroindustrial \\ Instituto Superior Tecnológico Juan Bautista Aguirre, Ecuador
}

\begin{abstract}
Good Syllabus content is a promise made by the teacher for the benefit of their students. For this reason, there is a need to assess the topics that are exposed in the different chairs, since the social, economic, and productive contexts are dynamic and evolve in a constant and sustained way. The research proposes a methodology for the evaluation and updating of the contents of the Syllabus, through 4 different perspectives: teacher, student, business, and trend, through the analysis of a case study applied to the subject of Applied Mathematics of the Technology in Administration degree and dictated during the II academic period 2020 - 2021 (December to May). The methodological proposal was developed by means of surveys to the specific focus groups (teachers, students, and business delegates), an analysis of the Syllabus of the subject of Mathematics Applied to Administration of the Bachelor of Administration of the National Technological Administration of Mexico and the contents of the Syllabus under analysis and that belonging to the Mexican institution were contrasted. Each of the 4 perspectives has a $25 \%$ percentage valuation in relation to the average of the averages in each of the items. The traffic lights of the results of the case study determined that $65 \%$ of the contents (items) should be kept within the Syllabus of Applied Mathematics of the Technology in Administration career, while $35 \%$ of said contents must be restructure, in such a way as to promote the application development of the contents within a labor-productive context. There are also thematic contents
\end{abstract}


that must be incorporated into the pedagogical document. The proposal is flexible, where the incorporation of other elements that help clarify and strengthen the methodology is possible.

Keywords: Evaluation, methodology, syllabus

\section{Introduction}

Syllabus proviene de la palabra latina Sillybus que significa enlistar o lista; en la actualidad se la utiliza para describir a todo documento que ayuda al docente a expresar de forma clara los objetivos y metas de la enseñanzaaprendizaje en un determinado curso. Dentro de los Syllabus se deben especificar los tópicos que serán abordados en cada ciclo de estudio, detallando las metodologías a aplicar y los mecanismos para la evaluación, de tal manera que los estudiantes conozcan el proceso efectivo de su enseñanzaaprendizaje que se llevará a cabo. Este documento actúa como un "contrato" implícito entre los docentes y los estudiantes, donde cada uno posee compromisos preestablecidos dentro del proceso, ya que el docente transmite contenidos temáticos y los estudiantes los interpreta y asimila (Caballero, 2013).

Se denominan diseños curriculares a la integración del macro, meso y microcurrículo. El macrocurrículo hace referencia al estudio de mercado y pertinencia de una carrera, mientras que el mesocurrículo se refiere al diseño curricular contextualizado para la misma carrera, por su parte, el microcurrículo es el detalle de los resultados de aprendizajes y los contenidos que abordarán las respectivas asignaturas (Méndez, Campi, Huayamave, \& Lozada, 2017). Bajo este contexto la presente investigación analizará uno de los principales aspectos curriculares, el microcurrículo o Syllabus.

Existe la noción de identificar de manera análoga al "currículo" y al "Syllabus", lo que a veces trae falsas interpretaciones en relación con estos dos instrumentos académicos. El currículo hace referencia a información general sobre los propósitos y experiencias de aprendizajes, la metodología de evaluación que se aplicará dentro de aula, además analiza los roles que desempeñarán los estudiantes y sus profesores durante el proceso de enseñanza-aprendizaje, finalmente este documento debe poseer apartados de las sugerencias metodológicas sobre la aplicación efectiva del currículo dentro del aula de clases. Por su parte, el Syllabus es más detallado, basándose en la selección y clasificación de los contenidos, analizando las actividades que se deben desarrollar para una efectiva interiorización de la información y aplicando los mecanismos de evaluación previamente establecidos (Carvajal \& Castaño, 2012).

Es necesario indicar que el perfil de egreso es la declaración de las instituciones educativas sobre los resultados de aprendizajes que alcanzarán 
los estudiantes al finalizar su malla escolar, estos nacen de la reflexión institucional de la demanda social en los ámbitos de desarrollo. También es considerada como una certificación de las competencias adquiridas durante sus fases de estudios, con el propósito de realizar una eficiente labor dentro de los espacios productivos. Por lo tanto, los perfiles de egresos orientan y delimitan a las instituciones sobre las áreas en las cuales deben girar sus programas educativos (Méndez, Bodero, Egüez, \& Plúa, 2020).

El Syllabus es una forma de comunicación escrita entre el docente, los estudiantes, los miembros de la comunidad educativa y los administradores. Para su cumplimiento es necesario conocer qué enseñar, cómo enseñar, cuándo enseñar, con qué recursos se dispone y cuáles serán los resultados que se desean alcanzar. Este documento es una planificación de los recursos disponibles, a través de una gestión dentro de los procesos pedagógicos, los cuales están enmarcados en tiempos de aplicación o períodos. Para que exista concordancia en el documento entre el perfil de egreso y las competencias propias de la carrera, el encargado en su realización debe conocer de forma prominente el área de estudio, de tal manera que se dimensione eficientemente los contenidos, el tiempo, los recursos, entre otros elementos. Para la estructura de un Syllabus, se deben considerar esquemas cerrados y abiertos, los primeros hacen referencia a los ejes gramaticales, las series de nociones, las series funcionales y al conjunto de actividades; por su parte, los abiertos se refieren a la selección de las tareas las cuales son organizadas con diferentes razonamientos críticos (Barros, Tapia, Chuchuca, \& Chuchuca, 2018).

La interpretación de los contenidos de los Syllabus o microcurrículos debe ser lógica y automática, los mismos deben referir información sobre el proceso de enseñanza-aprendizaje. Este documento dicta la ruta formativa que deberán cumplir los involucrados, siendo uno de los instrumentos necesarios para medir la calidad educativa (Álvarez \& Tabares, 2020). A pesar de la importancia que representa este documento, en muchas unidades educativas no se realiza el seguimiento y evaluación respectivo, por lo tanto, la información se encuentra desarticulada y desactualizada de su contexto social y productivo.

De manera general dentro de los Syllabus se encuentran los siguientes elementos: introducción de la asignatura, objetivos generales y específicos de la asignatura, resultados de aprendizajes a alcanzar, contenidos temáticos (divididos en unidades), metodologías propuestas para cada unidad de estudio, materiales a utilizar, mecanismos de evaluación y bibliografía. Para su estructuración se deben responder las siguientes preguntas: ¿Qué temas se van a abordar?, ¿Cómo se abordarán los tópicos?, ¿Qué recursos se deben emplear?, ¿Qué tiempo va a llevar alcanzar los resultados de aprendizajes propuestos? y ¿Qué se debe evaluar? (Matínez \& Rodríguez, 2014). 
La gestión curricular permite a los actores educativos el mapeo de los contenidos temáticos que se desarrollan dentro del aula, a través del conocimiento de sus fortalezas y debilidades, además consiente la posterior realización de su actualización. Esta evaluación temática debe responder a las siguientes preguntas ¿Quién lo hace? ¿Cómo se alinea con el perfil de egreso? ¿Con qué nivel de eficiencia y eficacia se trabaja? Este mapeo muestra la contribución vigente de los contenidos de cada una de las asignaturas en función del perfil de egreso de los estudiantes en cada carrera, además permite detectar aquellas competencias que deben ser potenciadas dentro del salón de clases, o aquellas que deben diseñarse en relación con las nuevas tendencias sociales o productivas (Icarte \& Labate, 2016).

Existen desfases en el currículo y el aporte de cada una de las asignaturas que lo componen. Esta desarticulación deriva de la tensión existente entre los plazos administrativos para ofertar un programa reformuladoy la premura de los representantes académicos para la realización de los perfiles de egreso, mallas, contenidos, etc. El resultado de ello es la existencia de programas académicos con competencias preestablecidas, que no están desarrolladas completamente en relación con el perfil de egreso "cambiable" que exigen las tendencias actuales. Esto trae consigo que exista un desarrollo no equilibrado en cada una de las competencias, donde cada asignatura otorga contenidos, pero no significados al hacer de la profesión. Por lo tanto, las instituciones educativas deben promover la revisión y modificación constante de sus contenidos curriculares, para que de manera articulada se desarrollen las competencias exigidas en los perfiles de egresos (Icarte \& Labate, 2016).

Dentro de los procesos de aseguramiento de la calidad educativa, las instituciones de educación superior deben desarrollar mecanismos que permitan conocer el estado de sus programas curriculares, estos diagnósticos consentirán la realización de un análisis interior crítico, con el objetivo de efectuar cambios pertinentes que logren la consecución de los objetivos institucionales o de la carrera. La denominada "libertad de cátedra" ha incorporado modelos de gestión y evaluación que únicamente operan en espacios institucionales internos, los cuales provocan tensiones en los procesos constructivos colectivos, que pueden representar un aporte cognitivo significativo para las instituciones que poseen menos recursos (Madrid, 2013). Los procesos de revisión de Syllabus representan una forma de manifestación del aseguramiento de la calidad en la educación superior, donde se vislumbran las necesidades formativas de los estudiantes, además faculta la realización de cambios en los contenidos o en las estrategias de la enseñanza. Si estos cambios son menores, con la actualización de los contenidos será suficiente, sin embargo, si los cambios son amplios, se debe realizar un rediseño de todo el currículo académico de la carrera (Icarte \& Labate, 2016). 
Como ya fue mencionado anteriormente, es necesario que las instituciones educativas realicen una evaluación interna de sus programas y contenidos temáticos, posteriormente se debe realizar una comparación entre instituciones que oferten planes similares, con el objetivo de establecer si los Syllabus realizados permiten resolver problemas en el mercado ocupacional, desde una cosmovisión diferente (Tinjacá \& Guerrero, 2018).

En el Reglamento de Régimen Académico, en su artículo 137, menciona que "El ajuste curricular es la modificación del currículo de una carrera o programa, que puede ser sustantivo o no sustantivo. Un ajuste curricular es sustantivo cuando modifica el objeto de estudio, objetivos de aprendizaje, perfil de egreso, tiempo de duración, cambio mayor al $25 \%$ de la modalidad de estudios, denominación de la carrera o programa, o denominación de la titulación. En tanto que, la modificación del resto de elementos del currículo, es de carácter no sustantivo. Las IES podrán realizar ajustes curriculares no sustantivos según sus procedimientos internos establecidos, los cuales deberán ser notificados al CES. Cuando las IES requieran realizar ajustes curriculares sustantivos deberán contar con la autorización del CES" (Consejo de Educación Superior, 2020). Esto indicaría que las Instituciones de Educación Superior, pueden realizar cambios no sustantivos en sus Syllabus, por lo tanto, surge la interrogante ¿Qué porcentajes de los contenidos de una asignatura pueden ser modificados, reemplazados o retirados?

Es necesario darle un valor agregado y diferenciador a los Syllabus, por lo tanto, estos deben ser evaluados de manera permanente, de tal manera, que exista una integración entre la planificación del documento y las acciones de quienes forman la comunidad educativa. Los Syllabus son el puente conector entre las nociones intangibles y los procesos tangibles, estos últimos lo realizan en primera instancia los docentes, pero a través de un proceso participativo puede incluirse a la sociedad a través de sus diferentes representantes y entes productivos (empresas públicas y privadas). Siempre existirán las preguntas ¿Cuáles son los contenidos útiles que deben estar en los Syllabus? o ¿Cuáles son los mecanismos para establecer si los contenidos en el Syllabus son de relevancia social, empresarial o productiva? La presente investigación tratará de abordar esta última interrogante, a través de la determinación de un modelo de propuesta metodológica para la evaluación y actualización de los contenidos de los Syllabus, en el contexto de la educación superior. Para ello, se realizan encuestas a docentes, estudiantes y personal empresarial en áreas administrativas, además se buscan paralelismos entre el Syllabus de la asignatura de Matemática Aplicada y el Syllabus de Matemática Aplicada a la Administración de la carrera de Licenciatura en Administración que oferta una institución de educación superior mexicana. 


\section{Metodología}

El enfoque de la investigación es mixto, donde se evidencian los elementos cualitativos en la determinación del semáforo de la escala cualitativa, siendo estas clasificadas en alto (verde), medio (amarillo) y nulo (rojo); por su parte, los elementos cuantitativos están presentes en la determinación de los porcentajes alcanzados para cada uno de los ítems en las diferentes encuestas realizadas. El diseño es de campo, debido a la realización de encuestas a: docentes afines a la asignatura de Matemática Aplicada, estudiantes del segundo nivel de la carrera de Tecnología en Administración ofertada por el Instituto Superior Tecnológico Juan Bautista Aguirre de la ciudad de Daule (Guayas - Ecuador), y representantes empresariales, los cuales desempeñan sus funciones en el área administrativa. También se realizó un análisis del nivel de compatibilidad con un Syllabus de una institución educativa que oferta una carrera con similares características. El alcance es descriptivo, ya que no se aborda la interrelación de causalidad y consecuencia, sino que se analiza una propuesta metodología para tratar de evaluar y actualizar los contenidos del Syllabus de la asignatura mencionada.

Para la determinación de las percepciones se la realizó lo siguiente:

- Docente: se aplicó una encuesta a 3 docentes que son afines a la asignatura de Matemática Aplicada.

- Estudiantil: se aplicó una encuesta a 19 estudiantes que cursaron la asignatura en análisis.

○ Empresarial: se solicitó autorización a 4 diferentes empresas para que sus delegados administrativos realicen la encuesta.

- Tendencial: únicamente se contrastaron los contenidos del Syllabus de Matemática Aplicada con los contenidos de Syllabus Matemática Aplicada a la Administración de la carrera de Licenciatura en Administración del Tecnológico Nacional de México.

Las encuestas permitieron conocer el nivel de aporte de los contenidos (ítems) al perfil de egreso, posteriormente se estimaron los porcentajes para cada uno de los ítems.

\section{Resultados}

La actual propuesta para la evaluación y actualización de los contenidos de los Syllabus de las carreras de educación superior se basa en las siguientes perspectivas: 


\section{Perspectivas para la evaluación y actualización del Syllabus}

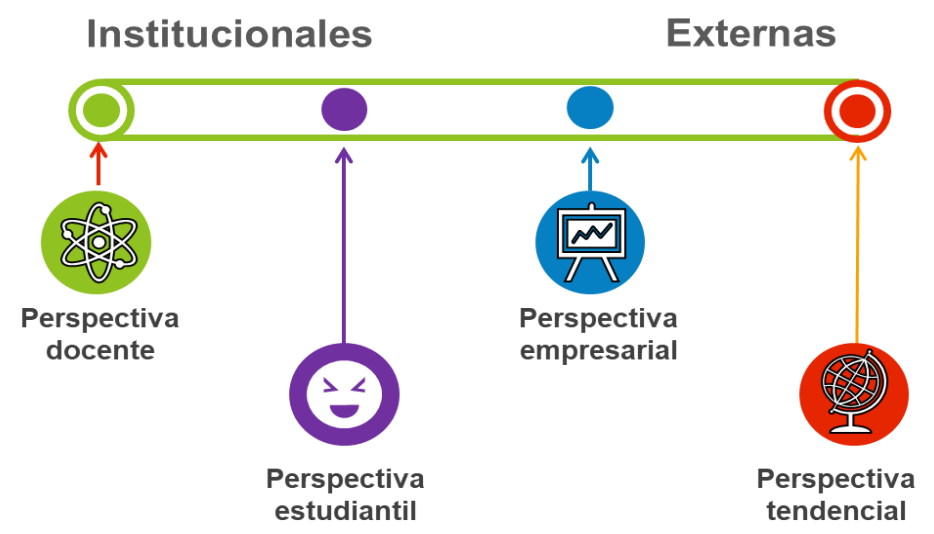

Figura 1. Perspectivas para la evaluación y actualización del Syllabus

Cada una de las perspectivas indica una visión y forma particular para percibir y evaluar los contenidos dentro de los Syllabus. Las perspectivas institucionales (docente y estudiantil) son aquellas que deben ejecutarse dentro del centro de estudios, por su parte, las externas (empresariales y tendenciales) se las ejecutan desde los espacios externos a la institución.

A continuación, se detallan las características de cada una de las perspectivas:

- Perspectiva docente: es aquella que deriva desde la visión que tienen los docentes sobre cómo una determinada asignatura aporta de manera significativa en el desarrollo de habilidades y competencias determinadas en el perfil de egreso.

- Perspectiva estudiantil: es aquella que deriva desde la visión que tienen los estudiantes sobre cómo una determinada asignatura aporta de manera significativa en el desarrollo de habilidades y competencias determinadas en el perfil de egreso.

- Perspectiva empresarial: es aquella que deriva desde la cosmovisión empresarial, productiva o institucional, donde los estudiantes realizan sus prácticas preprofesionales; en otras palabras, es la percepción de las entidades formativas sobre cómo las asignaturas que componen un plan de estudio aportan en el desarrollo de habilidades y competencias determinadas en el perfil de egreso.

- Perspectiva tendencial: es aquella que deriva del análisis de los contenidos en los Syllabus de otras carreras que guarden similitudes con el perfil de egreso y con sus asignaturas. El objetivo es contrastar y comparar ambos Syllabus y determinar el nivel de similitud. Estos comparativos ayudan a conocer los puntos de vistas de otras 
instituciones, adicionalmente permite proyectar contenidos que pueden añadirse al Syllabus.

Para validar la propuesta metodología de evaluación y actualización de los contenidos del Syllabus de la asignatura de Matemáticas Aplicada de la carrera e institución previamente mencionadas, se aplicaron encuestas a docentes, estudiantes, representantes empresariales y se comparó el Syllabus muestral con otro de una institución internacional, cuyos resultados de presentan a continuación:

\section{Perspectiva docente}

La encuesta fue aplicada a 3 docentes que dictaron la asignatura de Matemática Aplicada durante el II período académico 2020 - 2021 (diciembre a mayo). Cabe mencionar que esta asignatura fue desarrollada a través de plataformas digitales, debido a la implementación de la modalidad virtual de estudio por causa de la pandemia de covid-19. Los resultados obtenidos fueron los siguientes:

Tabla 1. Resultados de la perspectiva docente

\begin{tabular}{c|c}
\multicolumn{2}{c}{ Tabla 1. Resultados de la perspectiva docente } \\
\hline Encuesta para medir la perspectiva docente sobre los contenidos del Syllabus \\
\hline Carrera: & Tecnología en Administración \\
\hline Curso: & Segundo \\
\hline Muestra de análisis & 3 docentes \\
\hline
\end{tabular}

\section{Detalle del perfil de egreso:}

1. Emprender y evaluar ideas de negocios que promuevan la productividad y contribuyan al crecimiento eficaz de la empresa, ejerciendo las actividades con liderazgo y compromiso ético.

2. Comparar e interpretar la información financiera y económica, que contribuya a la toma de decisiones y permita generar oportunidades dentro de la empresa.

3. Aplicar métodos analíticos y estadísticos e interpretar sus resultados para una eficaz toma de decisiones en el contexto de la empresa.

4. Evaluar el impacto de las decisiones y acciones de la empresa que implica los recursos humanos como financieros para promover la equidad entre todos los integrantes de acuerdo con la naturaleza de la organización.

A continuación, se detallan los temas de las diferentes unidades que componen el Syllabus de la asignatura de "Matemáticas Aplicada". Desde su percepción, indicar con una "X" el nivel de aporte de los temas al desarrollo del perfil de egreso y a las competencias propias de la carrera.

\begin{tabular}{l|l|l|l}
\hline Ítem & Alto & Medio & Nulo \\
\hline Unidad 1: Aritmética y álgebra & $66,7 \%$ & $33,3 \%$ & \\
\hline l.1. Ecuaciones lineales & $66,7 \%$ & $33,3 \%$ & \\
\hline 1.2. Despeje de fórmulas & $66,7 \%$ & $33,3 \%$ & \\
\hline 1.3. Sistema de ecuaciones & $66,7 \%$ & $33,3 \%$ & \\
\hline 1.4. Aplicación a la Administración & $33,3 \%$ & $66,7 \%$ & \\
\hline 1.5. Ecuaciones cuadráticas & & $66,7 \%$ & $33,3 \%$ \\
\hline 1.6. Inecuaciones
\end{tabular}




\begin{tabular}{l|l|l|l}
\hline 1.7. Sistema de inecuaciones & $33,3 \%$ & $33,3 \%$ & $33,3 \%$ \\
\hline 1.8. Inecuaciones cuadráticas & & $66,7 \%$ & $33,3 \%$ \\
\hline Promedio & & & \\
\hline
\end{tabular}

Unidad 2: Limites y funciones

\begin{tabular}{l|l|l|l}
\hline 2.1. Matrices & $100 \%$ & & \\
\hline 2.2. Operaciones con matrices & $100 \%$ & & \\
\hline 2.3. Determinantes & $33,3 \%$ & $66,7 \%$ & \\
\hline 2.4. Funciones & $66,7 \%$ & $33,3 \%$ & \\
\hline 2.5. Tipos de funciones & $66,7 \%$ & $33,3 \%$ & \\
\hline 2.6. Dominio y recorrido de funciones & $66,7 \%$ & $33,3 \%$ & \\
\hline 2.7. Gráfico de una función & $66,7 \%$ & $33,3 \%$ & \\
\hline 2.8. Límites y propiedades & $33,3 \%$ & $33,3 \%$ & $33,3 \%$ \\
\hline 2.9. Características del cálculo diferencial & $33,3 \%$ & $33,3 \%$ & $33,3 \%$ \\
\hline Promedio & & & \\
\hline
\end{tabular}

En cada fila se detalla el porcentaje de los criterios de los participantes.

\section{Perspectiva estudiantil}

Está encuesta fue realizada a 19 estudiantes que cursaron la asignatura durante el II período académico 2020 - 2021 (diciembre a mayo). Los resultados obtenidos fueron los siguientes:

Tabla 2. Resultados de la perspectiva estudiantil

\begin{tabular}{c|c}
\hline \multicolumn{2}{c}{ Encuesta para medir la perspectiva estudiantil sobre los contenidos del Syllabus } \\
\hline Carrera: & Tecnología en Administración \\
\hline Curso: & Segundo \\
\hline Muestra de análisis & 19 estudiantes \\
\hline
\end{tabular}

Detalle del perfil de egreso:

1. Emprender y evaluar ideas de negocios que promuevan la productividad y contribuyan al crecimiento eficaz de la empresa, ejerciendo las actividades con liderazgo y compromiso ético.

2. Comparar e interpretar la información financiera y económica, que contribuya a la toma de decisiones y permita generar oportunidades dentro de la empresa.

3. Aplicar métodos analíticos y estadísticos e interpretar sus resultados para una eficaz toma de decisiones en el contexto de la empresa.

4. Evaluar el impacto de las decisiones y acciones de la empresa que implica los recursos humanos como financieros para promover la equidad entre todos los integrantes de acuerdo con la naturaleza de la organización.

A continuación, se detallan los temas de las diferentes unidades que componen el Syllabus de la asignatura de "Matemáticas Aplicada". Desde su percepción, indicar con una "X" el nivel de aporte de los temas al desarrollo del perfil de egreso y a las competencias propias de la carrera.

\begin{tabular}{l|l|l|l}
\hline Ítem & \multicolumn{1}{l|}{ Alto } & \multicolumn{1}{l}{ Medio } & Nulo \\
\hline Unidad 1: Aritmética y álgebra & $47,4 \%$ & $47,4 \%$ & $5,3 \%$ \\
\hline 1.1. Ecuaciones lineales & $47,4 \%$ & $47,4 \%$ & $5,3 \%$ \\
\hline 1.2. Despeje de fórmulas & $63,2 \%$ & $36,8 \%$ & \\
\hline 1.3. Sistema de ecuaciones & $63,2 \%$ & $31,6 \%$ & $5,3 \%$ \\
\hline 1.4. Aplicación a la Administración & $52,6 \%$ & $42,1 \%$ & $5,3 \%$ \\
\hline 1.5. Ecuaciones cuadráticas & $36,8 \%$ & $52,6 \%$ & $10,5 \%$ \\
\hline 1.6. Inecuaciones
\end{tabular}




\begin{tabular}{l|l|l|l}
\hline 1.7. Sistema de inecuaciones & $47,4 \%$ & $47,4 \%$ & $5,3 \%$ \\
\hline 1.8. Inecuaciones cuadráticas & $26,3 \%$ & $57,9 \%$ & $15,8 \%$ \\
\hline Promedio & \multicolumn{1}{l}{} \\
\hline Unidad 2: Límites y funciones & \multicolumn{1}{l}{} \\
\hline 2.1. Matrices & $42,1 \%$ & $57,9 \%$ & \\
\hline 2.2. Operaciones con matrices & $57,9 \%$ & $36,8 \%$ & $5,3 \%$ \\
\hline 2.3. Determinantes & $36,8 \%$ & $63,2 \%$ & \\
\hline 2.4. Funciones & $52,6 \%$ & $47,4 \%$ & \\
\hline 2.5. Tipos de funciones & $57,9 \%$ & $42,1 \%$ & \\
\hline 2.6. Dominio y recorrido de funciones & $47,4 \%$ & $47,4 \%$ & $5,3 \%$ \\
\hline 2.7. Gráfico de una función & $52,6 \%$ & $36,8 \%$ & $10,5 \%$ \\
\hline 2.8. Límites y propiedades & $31,6 \%$ & $57,9 \%$ & $10,5 \%$ \\
\hline 2.9. Características del cálculo diferencial & $31,6 \%$ & $52,6 \%$ & $15,8 \%$ \\
\hline Promedio & & & \\
\hline En cada fila se detalla el porcentaje de los criterios de los participantes. & \\
\hline
\end{tabular}

\section{Perspectiva empresarial}

El instrumento para la recolección de la información fue realizado a 4 representantes de diferentes sectores empresariales, quienes ejecutan sus actividades laborales en áreas afines a la administración y conocen las experticias que deben tener y desarrollar los futuros profesionales dentro de esta área. A continuación, el detalle de los resultados:

Tabla 3. Resultados de la perspectiva empresarial

\begin{tabular}{c|c}
\hline \multicolumn{2}{c}{ Encuesta para medir la perspectiva empresarial sobre los contenidos del Syllabus } \\
\hline Carrera: & Tecnología en Administración \\
\hline Curso: & Segundo \\
\hline Empresas: & Por confidencialidad no se detallan \\
\hline Muestra de análisis & 4 empresas \\
\hline
\end{tabular}

Detalle del perfil de egreso:

1. Emprender y evaluar ideas de negocios que promuevan la productividad y contribuyan al crecimiento eficaz de la empresa, ejerciendo las actividades con liderazgo y compromiso ético.

2. Comparar e interpretar la información financiera y económica, que contribuya a la toma de decisiones y permita generar oportunidades dentro de la empresa.

3. Aplicar métodos analíticos y estadísticos e interpretar sus resultados para una eficaz toma de decisiones en el contexto de la empresa.

4. Evaluar el impacto de las decisiones y acciones de la empresa que implica los recursos humanos como financieros para promover la equidad entre todos los integrantes de acuerdo con la naturaleza de la organización.

A continuación, se detallan los temas de las diferentes unidades que componen el Syllabus de la asignatura de "Matemáticas Aplicada". Desde su percepción, indicar con una "X" el nivel de aporte de los temas al desarrollo del perfil de egreso y a las competencias propias de la carrera.

\begin{tabular}{l|l|l|l}
\hline Ítem & Alto & Medio & Nulo \\
\hline Unidad 1: Aritmética y álgebra & $50 \%$ & $50 \%$ & \\
\hline 1.1. Ecuaciones lineales & $50 \%$ & $50 \%$ & \\
\hline 1.2. Despeje de fórmulas & $50 \%$ & $50 \%$ & \\
\hline 1.3. Sistema de ecuaciones &
\end{tabular}




\begin{tabular}{l|l|l|l}
\hline 1.4. Aplicación a la Administración & $75 \%$ & $25 \%$ & \\
\hline 1.5. Ecuaciones cuadráticas & $25 \%$ & $50 \%$ & $25 \%$ \\
\hline 1.6. Inecuaciones & $25 \%$ & $50 \%$ & $25 \%$ \\
\hline 1.7. Sistema de inecuaciones & $25 \%$ & $50 \%$ & $25 \%$ \\
\hline 1.8. Inecuaciones cuadráticas & $25 \%$ & $50 \%$ & $25 \%$ \\
\hline Promedio & \multicolumn{5}{l}{} \\
\hline Unidad 2: Límites y funciones & $25 \%$ & $50 \%$ & $25 \%$ \\
\hline 2.1. Matrices & $25 \%$ & $50 \%$ & $25 \%$ \\
\hline 2.2. Operaciones con matrices & & $50 \%$ & $50 \%$ \\
\hline 2.3. Determinantes & & $50 \%$ & $50 \%$ \\
\hline 2.4. Funciones & $50 \%$ & $50 \%$ \\
\hline 2.5. Tipos de funciones & $50 \%$ & $50 \%$ \\
\hline 2.6. Dominio y recorrido de funciones & $50 \%$ & $50 \%$ \\
\hline 2.7. Gráfico de una función & $50 \%$ & $50 \%$ \\
\hline 2.8. Límites y propiedades & $50 \%$ & \\
\hline 2.9. Características del cálculo diferencial & $50 \%$ & \\
\hline Promedio & \multicolumn{2}{l}{} \\
\hline En cada fila se detalla el porcentaje de los criterios de los participantes. & \\
\hline
\end{tabular}

\section{Perspectiva tendencial}

Para ejemplificar el modelo se seleccionó el Syllabus de Matemática Aplicada a la Administración del Tecnológico Nacional de México que se desarrolla en la carrera de Licenciatura en Administración. Se escogió este documento por las similitudes existentes en el perfil de egreso, además del reconocido prestigio de la mencionada institución. A nivel nacional los institutos públicos comparten los mismos modelos pedagógicos en todas las carreras que se ofertan en el país, por lo tanto, sería improcedente contrastar los contenidos entre instituciones de educación superior públicas. El modelo es flexible, esto indica que mientras existan similitudes en los perfiles de egreso y nombre de la carrera, sus contenidos pueden contrastarse entre sí. Los resultados obtenidos son los siguientes:

Tabla 4. Resultados de la perspectiva tendencial

Encuesta para medir la perspectiva tendencial sobre los contenidos del Syllabus

\begin{tabular}{|c|c|}
\hline $\begin{array}{l}\text { Nombre de la institución de la cual se } \\
\text { comparan los contenidos del Syllabus: }\end{array}$ & Tecnológico Nacional de México \\
\hline $\begin{array}{l}\text { País de la institución de la cual se } \\
\text { comparan los contenidos del Syllabus: }\end{array}$ & México \\
\hline $\begin{array}{l}\text { Nombre de la carrera de la cual se } \\
\text { comparan los contenidos del Syllabus: }\end{array}$ & Licenciatura en Administración \\
\hline $\begin{array}{l}\text { Nombre de la asignatura de la cual se } \\
\text { comparan los contenidos del Syllabus: }\end{array}$ & Matemática Aplicada a la Administración \\
\hline \multicolumn{2}{|c|}{$\begin{array}{l}\text { Link donde reposa el Syllabus comparado: } \\
\text { https://itescam.edu.mx/portal/asignatura.php?clave_asig=LAD-1027\&carrera=LADM- } \\
\text { 2010-234\&id_d=202 }\end{array}$} \\
\hline Nombre de la asignatura institucional: & Matemática Aplicada \\
\hline \multicolumn{2}{|c|}{ Detalle del Syllabus comparado } \\
\hline Unidad 1: Funciones maten & ticas y ecuaciones lineales \\
\hline
\end{tabular}


1.1. Definición

1.2. Dominio y rango restringidos

1.3. Funciones multivariadas básicas

1.4. Representaciones gráficas de funciones matemáticas

1.5. Fórmula pendiente intersección

1.6. Determinación de la ecuación de una línea recta

Unidad 2: Funciones lineales, aplicaciones y sistemas de ecuaciones lineales

2.1. Funciones lineales

2.2. Modelos de equilibrio

2.3. Sistemas de ecuaciones lineales

3.1. Introducción a las matrices

\section{Unidad 3: Álgebra matricial}

3.2. Tipos especiales de matrices

3.3. Operaciones con matrices

3.4. Introducción a los determinantes

3.5. Propiedades de los determinantes

3.6. Solución de la inversa de una matriz.

4.1. Limites y continuidad

Unidad 4: Diferenciación y Aplicaciones

4.2. Derivadas algebraicas con fórmulas

4.3. Derivada de n-ésimo orden

4.4. Derivadas parciales básicas

4.5. Aplicaciones de la primera y segunda derivada (a máximos y mínimos)

4.6. Aplicaciones a ingresos costos y utilidades

4.7. Análisis marginal

5.1. Concepto de antiderivada

\section{Unidad 5: Integración y Aplicaciones}

5.2. Reglas de integración directas

5.3. Integral definida

5.4. Aplicaciones del cálculo integral a problemas de las áreas administrativas

Indicar con una " $\mathrm{X}$ " los ítems que coinciden con los contenidos del Syllabus comparado

\begin{tabular}{l|l}
\hline Ítem & Nivel de coincidencia
\end{tabular}

\begin{tabular}{|c|c|c|c|}
\hline & Alto & Medio & Nulo \\
\hline \multicolumn{4}{|l|}{ Unidad 1: Aritmética y álgebra } \\
\hline 1.1. Ecuaciones lineales & $\mathrm{X}$ & & \\
\hline 1.2. Despeje de fórmulas & & $\mathrm{X}$ & \\
\hline 1.3. Sistema de ecuaciones & X & & \\
\hline 1.4. Aplicación a la Administración & $\mathrm{X}$ & & \\
\hline 1.5. Ecuaciones cuadráticas & & & $\mathrm{X}$ \\
\hline 1.6. Inecuaciones & & $\mathrm{X}$ & \\
\hline 1.7. Sistema de inecuaciones & & $\mathrm{X}$ & \\
\hline 1.8. Inecuaciones cuadráticas & & & $\mathrm{X}$ \\
\hline \multicolumn{4}{|l|}{ Unidad 2: Límites y funciones } \\
\hline 2.1. Matrices & $\mathrm{X}$ & & \\
\hline 2.2. Operaciones con matrices & $\mathrm{X}$ & & \\
\hline 2.3. Determinantes & $\mathrm{X}$ & & \\
\hline 2.4. Funciones & $\mathrm{X}$ & & \\
\hline 2.5. Tipos de funciones & $\mathrm{X}$ & & \\
\hline 2.6. Dominio y recorrido de funciones & $\mathrm{X}$ & & \\
\hline
\end{tabular}




\begin{tabular}{l|l|l|l}
\hline 2.7. Gráfico de una función & $\mathrm{X}$ & & \\
\hline 2.8. Límites y propiedades & $\mathrm{X}$ & & \\
\hline 2.9. Características del cálculo diferencial & $\mathrm{X}$ & & \\
\hline Porcentajes de coincidencias & $\mathbf{6 5 \%}$ & $20 \%$ & $\mathbf{1 5 \%}$ \\
\hline Detalle de contenidos que se podrían incorporar:
\end{tabular}

\section{Diferenciación y Aplicaciones}

Límites y continuidad

Derivadas algebraicas con fórmulas

Derivada de n-ésimo orden

Derivadas parciales básicas

Aplicaciones de la primera y segunda derivada (a máximos y mínimos)

Aplicaciones a ingresos costos y utilidades

Análisis marginal

Integración y Aplicaciones

Concepto de antiderivada

Reglas de integración directas

Integral definida

Aplicaciones del cálculo integral a problemas de las áreas administrativas

El porcentaje de coincidencias para cada nivel se realiza en base al total de coincidencias.

La comparación de los contenidos fue realizada por un grupo de docentes del Instituto Superior Tecnológico Juan Bautista Aguirre, los mismos que dictaron la cátedra en análisis. 


\section{Matriz de porcentajes finales}

Cada una de las perspectivas consideradas tiene una ponderación individual del $25 \%$. Los resultados coincidentes seleccionados en la perspectiva tendencial equivalen al $100 \%$. A continuación, los resultados finales:

\begin{tabular}{|c|c|c|c|c|c|c|c|c|c|c|c|c|c|c|c|}
\hline \multirow{3}{*}{ Ítem } & \multicolumn{6}{|c|}{ Perspectivas institucionales } & \multicolumn{6}{|c|}{ Perspectivas externas } & \multirow{2}{*}{\multicolumn{3}{|c|}{$\begin{array}{c}\text { Promedios de los } \\
\text { promedios }\end{array}$}} \\
\hline & \multicolumn{3}{|c|}{ Docente } & \multicolumn{3}{|c|}{ Estudiantil } & \multicolumn{3}{|c|}{ Empresarial } & \multicolumn{3}{|c|}{ Tendencial } & & & \\
\hline & Alto & Medio & Nulo & Alto & Medio & Nulo & Alto & Medio & Nulo & Alto & Medio & Nulo & Alto & Medio & Nulo \\
\hline U1.1.1. & $66,7 \%$ & $33,3 \%$ & & $47,4 \%$ & $47,4 \%$ & $5,3 \%$ & $50 \%$ & $50 \%$ & & $100 \%$ & & & $66 \%$ & $33 \%$ & $1 \%$ \\
\hline U1. 1.2. & $66,7 \%$ & $33,3 \%$ & & $47,4 \%$ & $47,4 \%$ & $5,3 \%$ & $50 \%$ & $50 \%$ & & & $100 \%$ & & $41 \%$ & $58 \%$ & $1 \%$ \\
\hline U1. 1.3. & $66,7 \%$ & $33,3 \%$ & & $63,2 \%$ & $36,8 \%$ & & $50 \%$ & $50 \%$ & & $100 \%$ & & & $70 \%$ & $30 \%$ & \\
\hline U1. 1.4. & $66,7 \%$ & $33,3 \%$ & & $63,2 \%$ & $31,6 \%$ & $5,3 \%$ & $75 \%$ & $25 \%$ & & $100 \%$ & & & $76 \%$ & $22 \%$ & $1 \%$ \\
\hline U1. 1.5 . & $33,3 \%$ & $66,7 \%$ & & $52,6 \%$ & $42,1 \%$ & $5,3 \%$ & $25 \%$ & $50 \%$ & $25 \%$ & & & $100 \%$ & $28 \%$ & $40 \%$ & $33 \%$ \\
\hline U1. 1.6. & & $66,7 \%$ & $33,3 \%$ & $36,8 \%$ & $52,6 \%$ & $10,5 \%$ & $25 \%$ & $50 \%$ & $25 \%$ & & $100 \%$ & & $15 \%$ & $67 \%$ & $17 \%$ \\
\hline U1. 1.7 . & $33,3 \%$ & $33,3 \%$ & $33,3 \%$ & $47,4 \%$ & $47,4 \%$ & $5,3 \%$ & $25 \%$ & $50 \%$ & $25 \%$ & & $100 \%$ & & $26 \%$ & $58 \%$ & $16 \%$ \\
\hline U1. 1.8. & & $66,7 \%$ & $33,3 \%$ & $26,3 \%$ & $57,9 \%$ & $15,8 \%$ & $25 \%$ & $50 \%$ & $25 \%$ & & & $100 \%$ & $13 \%$ & $44 \%$ & $44 \%$ \\
\hline U2. 2.1. & $100 \%$ & & & $42,1 \%$ & $57,9 \%$ & & $25 \%$ & $50 \%$ & $25 \%$ & $100 \%$ & & & $67 \%$ & $27 \%$ & $6 \%$ \\
\hline U2. 2.2. & $100 \%$ & & & $57,9 \%$ & $36,8 \%$ & $5,3 \%$ & $25 \%$ & $50 \%$ & $25 \%$ & $100 \%$ & & & $71 \%$ & $22 \%$ & $8 \%$ \\
\hline U2. 2.3. & $33,3 \%$ & $66,7 \%$ & & $36,8 \%$ & $63,2 \%$ & & & $50 \%$ & $50 \%$ & $100 \%$ & & & $43 \%$ & $45 \%$ & $13 \%$ \\
\hline U2. 2.4 . & $66,7 \%$ & $33,3 \%$ & & $52,6 \%$ & $47,4 \%$ & & & $50 \%$ & $50 \%$ & $100 \%$ & & & $55 \%$ & $33 \%$ & $13 \%$ \\
\hline U2. 2.5. & $66,7 \%$ & $33,3 \%$ & & $57,9 \%$ & $42,1 \%$ & & & $50 \%$ & $50 \%$ & $100 \%$ & & & $56 \%$ & $31 \%$ & $13 \%$ \\
\hline U2. 2.6. & $66,7 \%$ & $33,3 \%$ & & $47,4 \%$ & $47,4 \%$ & $5,3 \%$ & & $50 \%$ & $50 \%$ & $100 \%$ & & & $54 \%$ & $33 \%$ & $14 \%$ \\
\hline U2. 2.7. & $66,7 \%$ & $33,3 \%$ & & $52,6 \%$ & $36,8 \%$ & $10,5 \%$ & & $50 \%$ & $50 \%$ & $100 \%$ & & & $55 \%$ & $30 \%$ & $15 \%$ \\
\hline U2. 2.8 & $33,3 \%$ & $33,3 \%$ & $33,3 \%$ & $31,6 \%$ & $57,9 \%$ & $10,5 \%$ & & $50 \%$ & $50 \%$ & $100 \%$ & & & $41 \%$ & $35 \%$ & $23 \%$ \\
\hline U2. 2.9. & $33,3 \%$ & $33,3 \%$ & $33,3 \%$ & $31,6 \%$ & $52,6 \%$ & $15,8 \%$ & $50 \%$ & $50 \%$ & & $100 \%$ & & & $54 \%$ & $34 \%$ & $12 \%$ \\
\hline
\end{tabular}




\section{Semáforo de la escala cualitativa}

El porcentaje mayor del "promedio de los promedios" determinará las acciones por realizar, las cuales están contempladas en la siguiente manera:

Tabla 5. Semáforo de la escala cualitativa de los ítems del Syllabus

\begin{tabular}{c|c}
\hline Descripción & Medidas correctivas \\
\hline $\begin{array}{c}\text { Si el porcentaje mayor se encuentra en Alto } \\
\text { (verde) }\end{array}$ & El contenido del ítem se debe mantener \\
\hline $\begin{array}{c}\text { Si el porcentaje may se encuentra en Medio } \\
\text { (amarillo) }\end{array}$ & $\begin{array}{c}\text { El contenido del ítem se debe } \\
\text { reestructurar }\end{array}$ \\
\hline $\begin{array}{c}\text { Si el porcentaje mayor se encuentra en Nulo } \\
\text { (rojo) }\end{array}$ & $\begin{array}{c}\text { El contenido del ítem se debe } \\
\text { reemplazar }\end{array}$ \\
\hline
\end{tabular}

\section{Periodicidad de la evaluación}

La evaluación de los syllabus de parte de docentes y estudiantes debe realizarse al finalizar cada ciclo de estudios (períodos o semestres), mientras que el análisis de parte de las empresas debería realizarse al finalizar las prácticas preprofesionales de los estudiantes, por su parte, el análisis tendencial se debe ejecutar al finalizar la malla de una determinada cohorte. Por lo tanto, la evaluación general de un determinado ciclo será al finalizar la malla curricular de una determinada cohorte de estudiantes.

\section{Plasticidad muestral de la metodología}

La metodología tiene una alta flexibilidad para la determinación de cada una de las muestras, no obstante, se pueden considerar las siguientes recomendaciones:

- Muestra docente: se debe aplicar la encuesta a todo el cuerpo docente que tenga conocimiento del perfil de egreso de la carrera y del Syllabus de una determinada asignatura.

- Muestra estudiantil: las encuestas deben aplicarse por lo menos al 80 $\%$ de los estudiantes matriculados en la asignatura a analizar.

- Muestra empresarial: se debe realizar la encuesta en aquellas empresas en las cuales los estudiantes realizan sus prácticas preprofesionales, donde el número mínimo de empresas a encuestar no debe ser inferior a 3 .

- Muestra tendencial: el cuerpo docente debe analizar por lo menos 3 Syllabus de diferentes instituciones públicas o privadas, nacionales o internacionales. Ahondar en espacios internacionales permitirá conocer las tendencias en las cuales se desarrollan las carreras.

\section{Interpretación}

En base a los promedios establecidos obtenidos en cada uno de los ítems, se debe aplicar las siguientes medidas en cada uno de los contenidos: 


\begin{tabular}{l|c|c}
\hline Ítems & $\begin{array}{c}\text { Porcentaje } \\
\text { más alto }\end{array}$ & Medidas correctivas \\
\hline 1.1. Ecuaciones lineales & $66 \%$ & Mantener el contenido \\
\hline 1.2. Despeje de fórmulas & $58 \%$ & Reestructurar el contenido \\
\hline 1.3. Sistema de ecuaciones & $70 \%$ & Mantener el contenido \\
\hline 1.4. Aplicación a la Administración & $76 \%$ & Mantener el contenido \\
\hline 1.5. Ecuaciones cuadráticas & $40 \%$ & Reestructurar el contenido \\
\hline 1.6. Inecuaciones & $67 \%$ & Reestructurar el contenido \\
\hline 1.7. Sistema de inecuaciones & $58 \%$ & Reestructurar el contenido \\
\hline 1.8. Inecuaciones cuadráticas & $44 \%$ & Reestructurar el contenido \\
\hline 2.1. Matrices & $67 \%$ & Mantener el contenido \\
\hline 2.2. Operaciones con matrices & $71 \%$ & Mantener el contenido \\
\hline 2.3. Determinantes & $45 \%$ & Reestructurar el contenido \\
\hline 2.4. Funciones & $55 \%$ & Mantener el contenido \\
\hline 2.5. Tipos de funciones & $56 \%$ & Mantener el contenido \\
\hline 2.6. Dominio y recorrido de funciones & $54 \%$ & Mantener el contenido \\
\hline 2.7. Gráfico de una función & $55 \%$ & Mantener el contenido \\
\hline 2.8. Límites y propiedades & $41 \%$ & Mantener el contenido \\
\hline 2.9. Características del cálculo diferencial & $54 \%$ & Mantener el contenido \\
\hline
\end{tabular}

Según el análisis en la comparación de los Syllabus se deben añadir los siguientes contenidos:

○ Límites y continuidad

- Derivadas algebraicas con fórmulas

- Derivada de n-ésimo orden

- Derivadas parciales básicas

- Aplicaciones de la primera y segunda derivada (a máximos y mínimos)

- Aplicaciones a ingresos costos y utilidades

- Análisis marginal

- Concepto de antiderivada

- Reglas de integración directas

- Integral definida

- Aplicaciones del cálculo integral a problemas de las áreas administrativas

La reestructuración de los ítems debe ser entendida como un proceso en el cual se realiza la priorización de las aplicaciones prácticas de los contenidos sobre el desarrollo conceptual de los mismos. Si en una segunda evaluación, el mayor porcentaje se encuentra en la escala cualitativa de "medio", estos ítems deben ser reemplazados.

\section{Discusión}

La "radiografía" interna de los contenidos que poseen los Syllabus, ayuda a conocer si estos guardan relación directa con las perspectivas 
educativas que tienen los estudiantes, docentes y empresas. Por ello, debe existir una constante evaluación sobre la vigencia de sus contenidos, ya que las tendencias son dinámicas y demandan de actualizaciones en lapsos cortos de tiempo, lo que fomentaría al desarrollo de las competencias de los futuros profesionales.

Al no existir una metodología estandarizada para la evaluación de los Syllabus, es difícil realizar un análisis comparativo de los resultados encontrados, sin embargo, es posible determinar ciertos paralelismos con otras investigaciones que siguieron esta temática.

Cedeño (2015), menciona que existen varias problemáticas que dificultan la realización de los Syllabus, por ejemplo, la falta experiencia de los docentes en la construcción de estas herramientas pedagógicas, lo que provoca que existan disparidades entre los resultados de aprendizajes y el perfil de egreso. El análisis realizado en la anterior investigación fue de carácter cualitativo y se enfocó en el establecimiento del nivel de articulación de los contenidos temáticos en función de los trabajos autónomos y del soporte que estos dos elementos otorgan al perfil de egreso de los estudiantes.

Carvajal y Castaño (2012), realizaron la evaluación del Syllabus de la asignatura de Inglés dentro de una institución de educación primaria, quienes mencionaron que los objetivos deben ser claros, las herramientas precisas, los seguimientos adecuados, además los contenidos y actividades deben conducirse entre sí. También se indica que los propósitos de enseñanzaaprendizaje deben estar siempre definidos de manera clara, las pretensiones deben ser precisas en cuanto a lo que se enseña y para qué se enseña. En otras palabras, el enfoque desarrollado fue de manera global, no se abordaron detalles de la pertinencia de los contenidos específicos del Syllabus.

Idris (2018), realizó un análisis de los contenidos del Syllabus de la asignatura de Redacción de Informes, a través de un cuestionario cuantitativo que contenía 10 declaraciones para recopilar datos de los participantes. También se realizó una sesión de entrevistas con un conjunto de 5 preguntas basadas en los elementos claves del plan de estudios con los profesores para conocer su opinión experta y sugerencias. El análisis de los datos reveló que los contenidos eran pertinentes para los estudiantes y no necesita ser reemplazado. No obstante, se debían incluir algunas actividades que puedan abordar las necesidades de los estudiantes, finalmente se indica que los profesores que aprovechan otros recursos (de internet o de la biblioteca) otorgan un impulso profesionalizante adicional a los alumnos. Este último estudio tuvo elementos estadísticos similares a los aplicados en la presente investigación, los cuales respaldan la metodología aplicada.

Las investigaciones abordadas muestran particularidades diferentes para un mismo elemento (Syllabus), ya que fueron examinadas con diferentes metodologías pedagógicas, las cuales ayudan a puntualizar que los Syllabus 
deben ser evaluados a través de componentes cualitativos y cuantitativos. Al no existir una estandarización en el sistema de valoración siempre existirán muchas perspectivas, las cuales enriquecen el conocimiento de un elemento abstracto, que lejos de ser simplista es el eje principal que le da sentido y propósito al ejercicio docente.

\section{Conclusiones}

La propuesta metodología para la evaluación y actualización del Syllabus consiste en el análisis de las perspectivas docentes, estudiantiles, empresariales y tendenciales, donde cada una de ellas tiene la misma valoración porcentual en los resultados finales. Las perspectivas internas permiten conocer la pertinencia de los contenidos desde la visión institucional, mientras que las perspectivas externas logran determinar la viabilidad de los conocimientos en un plano laboral y de otras instituciones educativas, además consiente la incorporación de nuevos contenidos a los Syllabus.

De manera general, los mayores niveles de "nulidad" se obtuvieron de parte de las perspectivas estudiantiles y empresariales, pudiéndose interpretar que estos dos grupos perciben a los contenidos como desactualizados o inconsistentes de las nuevas tendencias. Esto también puede interpretarse como el efecto alienante que tiene el dogmatismo sistematizado de la educación sobre el profesorado, el cual, es incapaz de percibir desde adentro las incongruencias propias de régimen académico que lo gobierna y controla, olvidándose que es un ente reflexivo entodo el orbe educativo.

La propuesta es flexible y puede incorporar dimensiones adicionales, tales como el análisis de textos en el área de estudio, o entrevistas focalizadas a expertos. El objetivo final, es contar con una herramienta metodológica que permita desarrollar y fortalecer las competencias de los estudiantes, a través de un Syllabus que logre una triple articulación entre instituciones educativas, actores productivos y sociedad civil. Y aunque parezca utópico, la efectiva correspondencia de la educación a los retos contemporáneos consentirá el progreso de toda la sociedad.

\section{References:}

1. Álvarez, S., \& Tabares, N. (2020). Sistema de gestión de información del Syllabus -estudio de caso; Universidad Ces. Medellín, Antioquia, Colombia: Universidad CES. Obtenido de http://repository.ces.edu.co/handle/10946/4500

2. Barros, R., Tapia, S., Chuchuca, F., \& Chuchuca, I. (2018). Syllabus universitario actuante en ciencias pedagógicas potencialidades y limitaciones en la Universidad de Guayaquil. Revista Lasallista de Investigación, 15(2), 327-329. Obtenido de 
http://repository.lasallista.edu.co:8080/ojs/index.php/rldi/article/view/ 1872

3. Caballero, N. (2013). El Syllabus. El Tecnológico, 23(1), 22. Obtenido de https://revistas.utp.ac.pa/index.php/el-tecnologico/article/view/429

4. Carvajal, S., \& Castaño, J. (2012). Evaluación y mejoramiento del syllabus de inglés del grado de primero de primaria de un colegio monolíngue privado de Cali. Cali, Colombia: Universidad del Valle. Obtenido https://bibliotecadigital.univalle.edu.co/bitstream/handle/10893/9684/ CB0433798.pdf;jsessionid=CB023FF6DACEEB133E45DF13A9AF 0C3D? sequence $=1$

5. Cedeño, M. (2015). La acción dinamizadora de los Syllabus:una experiencia en construcción. Yachana, 4, 129-135. Obtenido de http://revistas.ulvr.edu.ec/index.php/yachana/article/view/144/105

6. Consejo de Educación Superior. (25 de agosto de 2020). Reglamento de Régimen Académico. Obtenido de https://procuraduria.utpl.edu.ec/sitios/documentos/NormativasPublica s/Reglamento\%20de\%20R\%C3\%A9gimen\%20Academico\%202020. pdf

7. Icarte, G., \& Labate, H. (2016). Metodología para la Revisión y Actualización de un Diseño Curricular de una Carrera Universitaria Incorporando Conceptos de Aprendizaje Basado en Competencias. Formación Universitaria, 9(2), 3-16. doi:10.4067/S071850062016000200002

8. Idris, S. (2018). An evaluation of technical report writing syllabus at the preparatory year program. Journal of English Education, 7(1), 1-8. Obtenido https://journal.uniku.ac.id/index.php/ERJEE/article/view/1528/1108

9. Madrid, R. (2013). El derecho a la libertad de cátedra y el concepto de universidad. Revista Chilena de Derecho, 40(1), 353-369. doi:10.4067/S0718-34372013000100016

10. Matínez, C., \& Rodríguez, L. (2014). Diseño e implementación de un Syllabus basados en contenidos y en el aprendizaje significativo para la enseñanza del Inglés en el segundo ciclo. Bogotá, Colombia: Universidad Libre. Obtenido de https://repository.unilibre.edu.co/bitstream/handle/10901/8219/Momi a\%20egipcia\%20elefante.pdf?sequence $=1 \&$ isAllowed $=\mathrm{y}$

11. Méndez, M., Bodero, K., Egüez, E., \& Plúa, R. (2020). Evaluación del perfil de egreso en la carrera de tecnología en procesamiento de alimentos en modalidad dual del Instituto Superior Tecnológico Juan Bautista Aguirre. Quito, Pichincha, Ecuador: Consejo de Aseguramiento de la Calidad de la Educación Superior. Obtenido de 
https://www.caces.gob.ec/wpcontent/uploads/Documents/PUBLICACIONES/CIFTEC\%202018\% 20LIBRO15_06_2020\%20(1).pdf

12. Méndez, M., Campi, I., Huayamave, Á., \& Lozada, A. (2017). Modelo de formación dual en el Instituto Tecnológico Superior "Juan Bautista". Ambato, Tungurahua, Ecuador: Pontificia Universidad Católica del Ecuador. Obtenido de https://repositorio.pucesa.edu.ec/handle/123456789/2309

13. Tinjacá, S., \& Guerrero, J. (2018). Hacia una nueva concepción del Syllabus Agustiniano. Bogotá, Colombia: Universidad Uniagustiniana. Obtenido de https://repositorio.uniagustiniana.edu.co/bitstream/handle/123456789 1469/TinjacaRivera-Santiago-2018.pdf?sequence $=4 \&$ isAllowed $=y$ 